\title{
Adhesive Categories
}

\author{
Stephen Lack $^{1}$ and Paweł Sobociński ${ }^{2}$ \\ 1 School of Quantitative Methods and Mathematical Sciences, \\ University of Western Sydney, \\ Australia \\ 2 BRICS $^{\star \star \star}$, University of Aarhus \\ Denmark
}

\begin{abstract}
We introduce adhesive categories, which are categories with structure ensuring that pushouts along monomorphisms are wellbehaved. Many types of graphical structures used in computer science are shown to be examples of adhesive categories. Double-pushout graph rewriting generalises well to rewriting on arbitrary adhesive categories.
\end{abstract}

\section{Introduction}

Recently there has been renewed interest in reasoning using graphical methods, particularly within the fields of mobility and distributed computing [19] as well as applications of semantic techniques in molecular biology 6,4. Research has also progressed on specific graphical models of computation [18. As the number of various models grows, it is important to understand the basic underlying principles of computation on graphical structures. Indeed, a solid understanding of the foundations of a general class of models (provided by adhesive categories, introduced in this paper), together with a collection of general semantic techniques (for example [21]) will provide practitioners and theoreticians alike with a toolbox of standard techniques with which to construct the models, define the semantics and derive proof-methods for reasoning about these.

Category theory provides uniform proofs and constructions across a wide range of models. The usual approach is to find a natural class of categories with the right structure to support the range of constructions particular to the application area. A well-known example is the class of cartesian-closed categories, which provides models for simply typed lambda calculi [17].

In this paper we shall demonstrate that adhesive categories have structure which allows a development of a rich general theory of double-pushout (d-p) rewriting [13. D-p graph rewriting has been widely studied and the field can be considered relatively mature 201812.

In D-p rewriting, a rewrite rule is given as a span $L \leftarrow K \rightarrow R$. Roughly, the intuition is that $L$ forms the left-hand side of the rewrite rule, $R$ forms the right-hand side and $K$, common to both $L$ and $R$, is the sub-structure to be unchanged as the rule is applied. To apply the rule to a structure $C$, one

\footnotetext{
$\star \star \star$ Basic Research in Computer Science (www.brics.dk)
} funded by the Danish National Research Foundation. 
first needs to find a match $L \rightarrow C$ of $L$ within $C$. The rule is then applied by constructing the missing parts $(E, D$ and arrows) of the following diagram

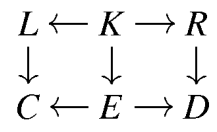

in a way which ensures that the two squares are pushout diagrams. Once such a diagram is constructed we may deduce that $C \longrightarrow \square D$, that is, $C$ rewrites to $D$.

D-p rewriting is formulated in categorical terms and is therefore portable to structures other than directed graphs. There have been several attempts [119] to isolate classes of categories in which one can perform d-p rewriting and in which one can develop the rewriting theory to a satisfactory level. In particular, several axioms were put forward in [1] in order to prove a local Church-Rosser theorem for such general rewrite systems. Additional axioms were needed to prove a general version of the so-called concurrency theorem [14.

An important general construction which appears in much of the literature on graphical structures in computer science is the pushout construction. Sometimes referred to as generalised union [9], it can often be thought of as the construction of a larger structure from two smaller structures by gluing them together along a shared substructure.

One can think of adhesive categories as categories in which pushouts along monomorphisms are "well-behaved", where the paradigm for behaviour is given by the category of sets. An example of the good behaviour of these pushouts is that they are stable under pullback (the dual notion to pushout, which intuitively can often be thought of as a "generalised intersection"). The idea is analogous to that of extensive categories 3], which have well-behaved coproducts in a similar sense. Since coproducts can be obtained with pushouts and an initial object, and an initial object is "well-behaved" if it is strict, one might expect that adhesive categories with a strict initial object would be extensive, and this indeed turns out to be the case.

Various notions of graphical structures used in computer science form adhesive categories. This includes ordinary directed graphs, typed graphs [1] and hypergraphs [1], amongst others. The structure of adhesive category allows us to derive useful properties. For instance, the union of two subobjects is calculated as the pushout over their intersection, which corresponds well with the intuition of pushout as generalised union.

We shall consider adhesive grammars which are d-p rewrite systems on adhesive categories. We show that the resulting rewriting theory is satisfactory by proving the local Church-Rosser theorem and the concurrency theorem without the need for extra axioms. We shall also examine how adhesive categories fit within the previously conceived general frameworks for rewriting [11|9]. Many of the axioms put forward in [11] follow elegantly as lemmas from the axioms of adhesive categories.

Adhesive categories, therefore, provide a satisfactory model in which to define a theory of rewriting on "graph-like" structures. They are mathematically elegant and arguably less ad-hoc than previous approaches. We firmly believe 
that they will prove useful in the development of further theory in the area of semantics of graph-based computation, and in particular, in the development of a contextual theory of graph rewriting.

Structure of the paper. In $\$$ we recall the definition of extensive categories. The notion of van Kampen (VK) square is given in $\$ 2$ VK squares are central in the definition of adhesive categories which are introduced in $\$ 3$. In \$4 we state and prove some basic lemmas which hold in any adhesive category. We also show that the subobjects of an object in an adhesive category form a distributive lattice, with the union of two subobjects constructed as the pushout over their intersection. We develop double-pushout rewriting theory in adhesive categories in \$5 and offer a comparison with High-Level Replacement Categories in \$6. We conclude in $\$ 7$ with directions for future research.

Many of the proofs have been omitted. The interested reader may wish to consult the full version [15].

\section{Extensive Categories}

Throughout the paper we assume that the reader is familiar with basic concepts of category theory. In this section we recall briefly the notion of extensive category [3].

Definition $\mathbf{1}$ A category $\mathbf{C}$ is said to be extensive when

(i) it has finite coproducts

(ii) it has pullbacks along coproduct injections

(iii) given a diagram where the bottom row is a coproduct diagram

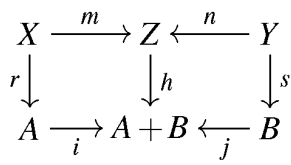

the two squares are pullbacks if and only if the top row is a coproduct.

The third axiom states what we mean when we say that the coproduct $A+B$ is "well-behaved": it includes the fact that coproducts are stable under pullback, and it implies that coproducts are disjoint (the pullback of the coproduct injections is initial) and that initial objects are strict (any arrow to an initial object must be an isomorphism). It also implies a cancellativity property of coproducts: given an isomorphism $A+B \cong A+C$ compatible with the injections, one can construct an isomorphism $B \cong C$. For an object $Z$ of an extensive category, the lattice $\operatorname{Sub}(Z)$ of coproduct summands of $Z$ is a Boolean algebra.

\section{Van Kampen Squares}

The definition of adhesive category is stated in terms of something called a van Kampen square, which can be thought of as a "well-behaved pushout", in 
a similar way to which coproducts can be thought of as "well-behaved" in an extensive category; essentially this means that they behave as they do in the category of sets.

The name van Kampen derives from the relationship between these squares and the van Kampen theorem in topology, in its "coverings version", as presented for example in [2]. This relationship is described in detail in [16].

Definition 2 (van Kampen square) A van Kampen (VK) square (i) is a pushout which satisfies the following condition: given a commutative cube (ii) of which ( $i$ ) forms the bottom face and the back faces are pullbacks,

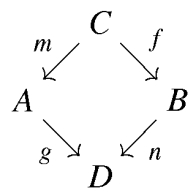

(i)

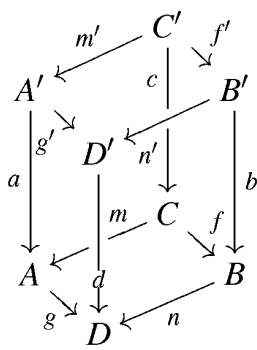

(ii)

the front faces are pullbacks if and only if the top face is a pushout. Another way of stating the "only if" condition is that such a pushout is required to be stable under pullback.

Another, equivalent, way of defining a VK square in a category with pullbacks is as follows. A VK square (i) is a pushout which satisfies the property that given a commutative diagram (iii), the two squares are pullbacks if and only if there exists an object $C^{\prime}$ and morphisms

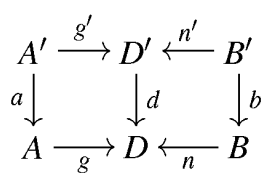

(iii)

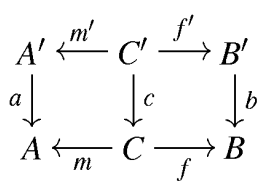

(iv)

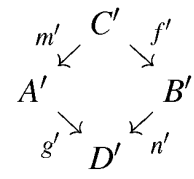

$(v)$

so that the squares in (iv) are pullbacks and (v) is a pushout.

By a pushout along a monomorphism we mean a pushout, as in Diagram (i) above, in which $m$ is a monomorphism. Similarly, if $m$ is a coproduct injection, we have a pushout along a coproduct injection.

A crucial class of examples of VK squares is provided by:

Theorem 3. In an extensive category, pushouts along coproduct injections are VK squares.

We have the following important properties of VK squares: 
Lemma 4 In a VK square as in (i), if $m$ is a monomorphism then $n$ is a monomorphism and the square is also a pullback.

Proof. Suppose that the bottom face of the cube

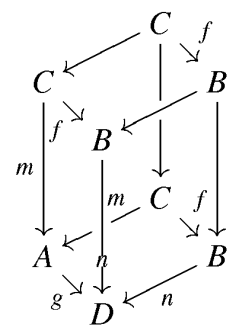

is VK. Then the top and bottom squares are pushouts, while the back squares are pullbacks if $m$ is a monomorphism. Thus the front faces will be pullbacks: the front right face being a pullback means that $n$ is a monomorphism, and the front left face being a pullback means that the original square is a pullback.

\section{Adhesive Categories}

We shall now proceed to define the notion of adhesive category, and provide various examples and counterexamples.

Definition 5 (Adhesive category) A category $\mathbf{C}$ is said to be adhesive if

(i) C has pushouts along monomorphisms;

(ii) C has pullbacks;

(iii) pushouts along monomorphisms are VK-squares.

Just as the third axiom of extensive categories (Definition 1) ensures that coproducts are "well-behaved", it is the third axiom of adhesive categories which ensures that pushouts along monomorphisms are "well-behaved". This includes the fact that such pushouts are stable under pullback.

Since every monomorphism in Set is a coproduct injection, and Set is extensive, we immediately have:

Example 6 Set is adhesive.

Observe that the restriction to pushouts along monomorphisms is necessary: there are pushouts in Set which are not VK squares. Consider the 2 element abelian group $\mathbb{Z}_{2}$ (the following argument works for any non-trivial group). In the diagram

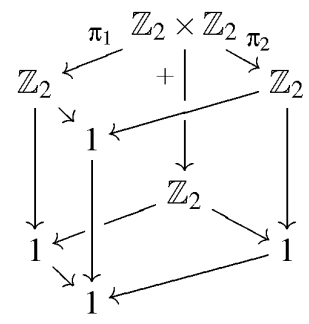


both the bottom and the top faces are easily verified to be pushouts and the rear faces are both pullbacks. However, the front two faces are not pullbacks.

Even with the restriction to pushouts along a monomorphism, many wellknown categories fail to be adhesive.

Counterexample 7 The categories Pos, Top, Gpd and Cat are not adhesive.

Since the definition of adhesive category only uses pullbacks, pushouts, and relationships between these, we have the following constructions involving adhesive categories:

\section{Proposition 8}

(i) If $\mathbf{C}$ and $\mathbf{D}$ are adhesive categories then so is $\mathbf{C} \times \mathbf{D}$;

(ii) If $\mathbf{C}$ is adhesive then so are $\mathbf{C} / C$ and $C / \mathbf{C}$ for any object $C$ of $\mathbf{C}$;

(iii) If $\mathbf{C}$ is adhesive then so is any functor category $[\mathbf{X}, \mathbf{C}]$.

Since Set is adhesive, part (iii) of the proposition implies that any presheaf topos $[\mathbf{X}, \mathbf{S e t}]$ is adhesive. In particular, the category Graph of directed graphs is adhesive. Indeed, if $\mathbf{C}$ is adhesive, then so is the category $\operatorname{Graph}(\mathbf{C})=[\cdot \rightrightarrows$ $\cdot, \mathbf{C}]$ of internal graphs in $\mathbf{C}$.

Part (ii) implies that categories of typed graphs 1], coloured (or labelled) graphs [5] and hypergraphs [1], considered in the literature on graph grammars, are adhesive.

As a consequence, all proof techniques and constructions in adhesive categories can be readily applied to any of the aforementioned categories of graphs. In fact, more generally, we have:

Proposition 9 Any elementary topos is adhesive.

This is somewhat harder to prove than the result for presheaf toposes; the proof can be found in [16].

Part (ii) of Proposition 8 also allows us to construct examples of adhesive categories which are not toposes.

Example 10 The category Set $_{*}=1 /$ Set of pointed sets (or equivalently, sets and partial functions) is adhesive, but is not extensive, and therefore, is not a topos.

\section{Basic Properties of Adhesive Categories}

Here we provide several simple lemmas which hold in any adhesive category. Lemma 11] demonstrates why adhesive categories can be considered as a generalisation of extensive categories. Lemmas [12] [13, 15] and [16] shed some light on pushouts in adhesive categories.

Lemma 11 An adhesive category is extensive if and only if it has a strict initial object. 
Proof. In an extensive category the initial object is strict [3, Proposition 2.8]. On the other hand, in an adhesive category with strict initial object, any arrow with domain 0 is mono. Consider the cube

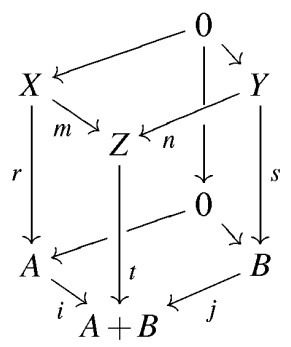

in which the bottom square is a pushout along a monomorphism, while the back squares are pullbacks since the initial object is strict. By adhesiveness, front squares are pullbacks if and only if the top squares is a pushout; but this says that the front squares are pullbacks if and only if the top row of these squares is a coproduct $(\mathrm{Z}=\mathrm{X}+\mathrm{Y})$.

The conclusions of the following two lemmas are used extensively in literature on algebraic graph rewriting. Indeed, they are usually assumed as axioms (see [9] and below) in attempts at generalising graph rewriting. They hold in any adhesive category by Lemma 4:

Lemma 12 Monomorphisms are stable under pushout.

Lemma 13 Pushouts along monomorphisms are also pullbacks.

The notion of pushout complement 13 is vital in algebraic approaches to graph rewriting.

Definition 14 Let $m: C \rightarrow A$ and $g: A \rightarrow B$ be arrows in an arbitrary category ( $m$ is not assumed to be mono). A pushout complement of the pair $(m, g)$ consists of arrows $f: C \rightarrow B$ and $n: B \rightarrow D$ for which the resulting square commutes and is a pushout. We shall sometimes refer to pushout complements of monos, this refers to pushout complements of pairs $(m, g)$ where $m$ is mono.

The conclusion of the following lemma is a crucial ingredient in many applications of graph rewriting. It has also been assumed as an axiom [11] in order to prove the concurrency theorem (cf. Theorem 27). It is important mainly because it assures that once an occurrence of a left hand side of a rewrite rule is found within a structure, then the application of the rewrite rule results in a structure which is unique up to isomorphism (cf. \$5). In other words, rewrite rule application is functional up to isomorphism.

Lemma 15 Pushout complements of monos (if they exist) are unique up to isomorphism. 
Proof. Suppose that the following diagrams

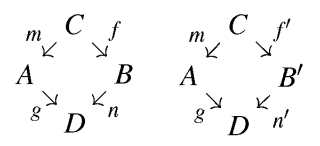

are pushouts and that $m$ is mono. Consider the cube

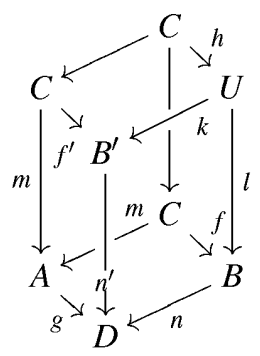

in which the front right face is a pullback, $h: C \rightarrow U$ is the map induced by $f$ and $f^{\prime}$, and the unnamed arrows are identities. Then the front faces and the back left face are pullbacks, hence the back right face is also a pullback; and the bottom face is a pushout, hence the top face is a pushout. But this implies that $k$ is invertible, since it is the pushout of $1_{C}$. By symmetry, so too is $l$. The induced isomorphism $j=k l^{-1}: B \rightarrow B^{\prime}$ satisfies $n^{\prime} j=n$ and $j f=f^{\prime}$.

The final lemma of this section will be used in Section 6 to show that adhesive categories are high-level replacement categories:

Lemma 16 Consider a diagram

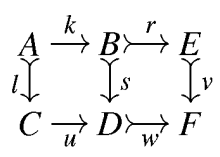

in which the marked morphisms are mono, the exterior is a pushout and the right square is a pullback. Then the left square is a pushout, and so all squares are both pullbacks and pushouts.

Proof. This amounts to stability of the exterior pushout under pullback along $w: D \rightarrow F$.

\subsection{Algebra of Subobjects}

We can put a preorder on monomorphisms into an object $Z$ of an arbitrary category by defining a monomorphism $a: A \rightarrow Z$ to be less than or equal to a monomorphism $b: B \rightarrow Z$ precisely when there exists an arrow $c: A \rightarrow B$ such that $b c=a$. A subobject (of $Z$ ) is an equivalence class with respect to 
the equivalence generated by this preorder. For example, subobjects in Set are subsets while subobjects in Graph are subgraphs.

Here we shall demonstrate that, in adhesive categories, unions of two subobjects can be constructed by pushout over their intersection. This provides further evidence of how pushouts behave in adhesive categories as well as making more precise the intuition that the pushout operation "glues together" two structures along a common substructure. As a corollary, it follows that in an adhesive category the lattices of subobjects are distributive.

Let $\mathbf{C}$ be an adhesive category, and $Z$ a fixed object of $\mathbf{C}$. We write $\operatorname{Sub}(Z)$ for the category of subobjects of $Z$ in $\mathbf{C}$; it has products (=intersections), given by pullback in $\mathbf{C}$. It has a top object, given by $Z$ itself. If $\mathbf{C}$ has a strict initial object 0 , then the unique map $0 \rightarrow Z$ is a monomorphism, and is the bottom object of $\operatorname{Sub}(Z)$.

Theorem 17. For an object $Z$ of an adhesive category $\mathbf{C}$, the category $\operatorname{Sub}(Z)$ of subobjects of $Z$ has binary coproducts: the coproduct of two subobjects is the pushout in $\mathbf{C}$ of their intersection.

Since pushouts are stable it follows that intersections distribute over unions:

Corollary 18 The lattice $\operatorname{Sub}(Z)$ is distributive.

\section{Double-Pushout Rewriting}

Here we shall recall the basic notions of double-pushout rewriting [1320] and show that it can be defined within an arbitrary adhesive category.

Henceforth we shall assume that $\mathbf{C}$ is an adhesive category.

Definition 19 (Production) A production $p$ is a span

$$
L \stackrel{l}{\leftarrow} K \stackrel{r}{\rightarrow} R
$$

in $\mathbf{C}$. We shall say that $p$ is left-linear when $l$ is mono, and linear when both $l$ and $r$ are mono. We shall let $\mathcal{P}$ denote an arbitrary set of productions and let $p$ range over $\mathcal{P}$.

In order to develop an intuition of why a production is defined as a span, we shall restrict our attention to linear production rules. One may then consider $K$ as a substructure of both $L$ and $R$. We think of $L$ and $R$ as respectively the left-hand side and the right-hand side of the rewrite rule $p$. In order to perform the rewrite, we need to match $L$ as a substructure of a redex $C$. The structure $K$, thought of as a substructure of $L$, is exactly the part of $L$ which is to remain invariant as we apply the rule to $C$.

Thus, an application of a rewrite rule consists of three steps. First we must match $L$ as a substructure of the redex $C$; secondly, we delete all of parts of the redex matched by $L$ which are not included in $K$. Thirdly, we add all of $R$ which is not contained in $K$, thereby producing a new structure $D$. The deletion and addition of structure is handled, respectively, by finding a pushout complement and constructing a pushout. 
Definition 20 (Gluing Conditions) Given a production $p$ as in (1), a match in $C$ is a morphism $f: L \rightarrow C$. A match $f$ satisfies the gluing conditions with respect to $p$ precisely when there exists an object $E$ and morphisms $g: K \rightarrow E$ and $v: E \rightarrow C$ such that

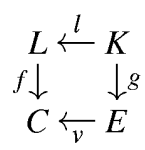

is a pushout diagram. (In other words, there exists a pushout complement of $(l, f)$ in the sense of Definition[14.)

Definition 21 (Derivation) Given an object $C \in \mathbf{C}$ and a set of productions $\mathcal{P}$, we write $C \longrightarrow \triangleright_{p, f} D$ for a production $p \in \mathcal{P}$ and a morphism $f: L \rightarrow C$ if (a) $f$ satisfies the gluing conditions with respect to $l$, and (b) there is a diagram

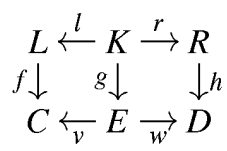

in which both squares are pushouts.

The object $E$ in the above diagram can be thought of as a temporary state in the middle of the rewrite process. Returning briefly to our informal description, it is the structure obtained from $C$ by deleting all the parts of $L$ not contained in $K$. Recall from Lemma 15 that if $l$ is mono (that is, if $p$ is left-linear) then $E$ is unique up to isomorphism. Indeed, if $p$ is a left-linear production, $C \longrightarrow \triangleright_{p, f} D$ and $C \longrightarrow \triangleright_{p, f} D^{\prime}$ then we must have $D \cong D^{\prime}$. This is a consequence of Lemma 15 and the fact that pushouts are unique up to isomorphism.

Definition 22 (Adhesive Grammar) An adhesive grammar $\mathbf{G}$ is a pair $\langle\mathbf{C}, \mathbf{P}\rangle$ where $\mathbf{C}$ is an adhesive category and $\mathbf{P}$ is a set of linear productions.

Assuming that all the productions are linear allows us to derive a rich rewriting theory on adhesive categories. Henceforward we assume that we are working over an adhesive grammar $\mathbf{G}$.

\subsection{Local Church-Rosser Theorem}

As shall be explained in section [6 adhesive categories with coproducts are highlevel replacement categories. In particular, we get the local Church-Rosser theorem 149.

Before presenting this theorem we need to recall briefly the notions of parallelindependent derivation and sequential-independent derivation. The reader may wish to consult [5] for a more complete presentation.

A parallel-independent derivation is a pair of derivations

$$
C \longrightarrow \triangleright_{p_{1}, f_{1}} D_{1} \quad \text { and } \quad C \longrightarrow \triangleright_{p_{2}, f_{2}} D_{2}
$$


as illustrated in diagram (5.1) which satisfy an additional requirement, namely the existence of morphisms $r: L_{1} \rightarrow E_{2}$ and $s: L_{2} \rightarrow E_{1}$ which render the diagram commutative, in the sense that $v_{2} r=f_{1}$ and $v_{1} s=f_{2}$.

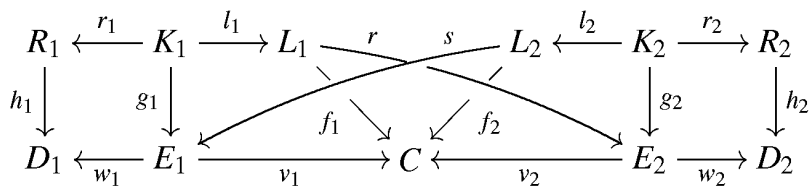

Similarly, a sequential-independent derivation, illustrated in diagram (5.1), is a derivation

$$
C \longrightarrow \triangleright_{p_{1}, f_{1}} D_{1} \longrightarrow \triangleright_{p_{2}, f_{2}^{\prime}} D
$$

where there additionally exist arrows $r^{\prime}: R_{1} \rightarrow E_{3}$ and $s^{\prime}: L_{2} \rightarrow E_{1}$ such that $w_{1} s^{\prime}=f_{2}^{\prime}$ and $v_{3} r^{\prime}=h_{1}$.

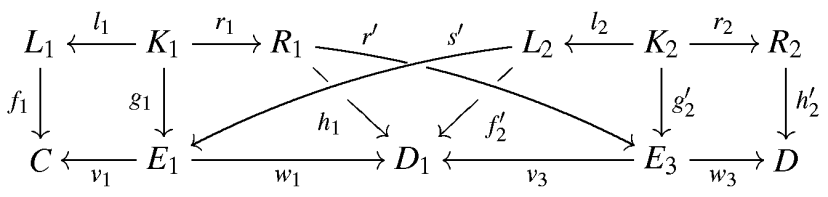

The statement of the theorem below differs from those previously published in the literature in that we do not need coproducts to establish the equivalence of the first 3 items.

Theorem 23 (Local Church-Rosser). The following are equivalent

1. $C \longrightarrow \triangleright_{p_{1}, f_{1}} D_{1}$ and $C \longrightarrow \triangleright_{p_{2}, f_{2}} D_{2}$ are parallel-independent derivations

2. $C \longrightarrow \triangleright_{p_{1}, f_{1}} D_{1}$ and $D_{1} \longrightarrow \triangleright_{p_{2}, f_{2}^{\prime}} D$ are sequential-independent derivations

3. $C \longrightarrow \triangleright_{p_{2}, f_{2}} D_{2}$ and $D_{2} \longrightarrow \triangleright_{p_{1}, f_{1}^{\prime}} D$ are sequential-independent derivations.

If moreover $\mathbf{C}$ is extensive then we may add the so-called parallelism theorem

4. $C \longrightarrow \triangleright_{p_{1}+p_{2},\left[f_{1}, f_{2}\right]} D$ is a derivation.

In fact, the proof that $(1) \Rightarrow(2)$ remains valid more generally in the context of left-linear productions, but the proof of the converse requires linearity.

\subsection{Concurrency Theorem}

The original concurrency theorems were proved for graph grammars [7] and later generalised to high-level replacement categories (cf. 86) in [11] which satisfy additional axiom sets, there called HLR2 and HLR2*. Roughly, the concurrency theorem states that given two derivations in a sequence, together with information about how they are related, one may construct a single derivation which internalises the two original derivations and performs them "concurrently". Moreover, one may reverse this process and deconstruct a concurrent derivation into two 
related sequential derivations. Here we state and prove the concurrency theorem for adhesive grammars without the need for extra axioms.

We shall first need to recall the notions of dependency relation, dependent derivation and concurrent production.

Definition 24 (Dependency Relation) Suppose that $p_{1}$ and $p_{2}$ are linear productions. A dependency relation for $\left\langle p_{1}, p_{2}\right\rangle$ is an object $X$ together with arrows $s: X \rightarrow R_{1}$ and $t: X \rightarrow L_{2}$ for which $r_{1}, s, t$, and $l_{2}$ can be incorporated into a diagram

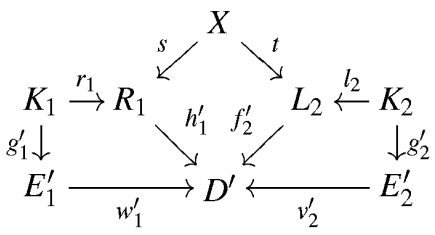

in which all three regions are pushouts.

Definition 25 (Dependent Derivation) Consider a derivation $C-\triangleright_{p_{1}, f_{1}}$ $D_{1} \longrightarrow \triangleright_{p_{2}, f_{2}} D$ as illustrated in (i) below

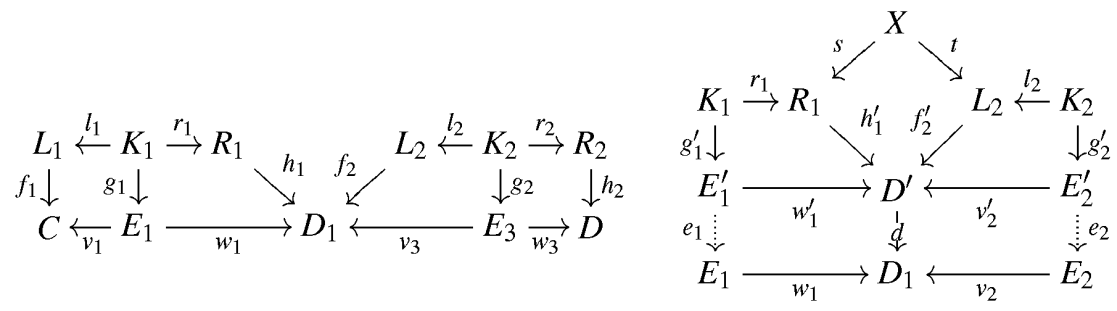

(i)

(ii)

and a dependency relation $X$ for $\left\langle p_{1}, p_{2}\right\rangle$. The derivation is said to be $X$ dependent if $h_{1} s=f_{2} t$ and there exist morphisms $e_{1}: E_{1}^{\prime} \rightarrow E_{1}$ and $e_{2}: E_{2}^{\prime} \rightarrow E_{2}$ satisfying $e_{1} g_{1}^{\prime}=g_{1}$ and $e_{2} g_{2}^{\prime}=g_{2}$, and if moreover the unique map $d: D^{\prime} \rightarrow D_{1}$ satisfying $d h_{1}^{\prime}=h_{1}$ and $d f_{2}^{\prime}=f_{2}$ also satisfies $d w_{1}^{\prime}=w_{1} e_{1}$ and $d v_{2}^{\prime}=v_{2} e_{2}$ (see (ii)).

Definition 26 (Concurrent Production) Given a dependency relation $X$ for $\left\langle p_{1}, p_{2}\right\rangle$, the $X$-concurrent production $p_{1} ; x p_{2}$ is the span

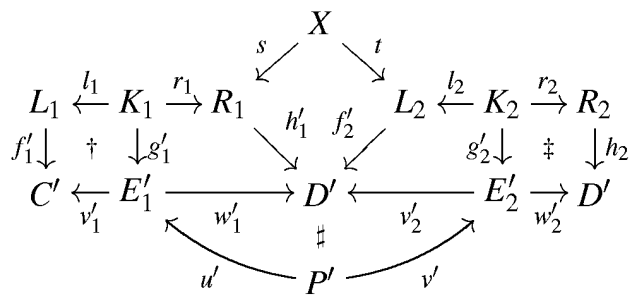

in which $\dagger$ and $\ddagger$ are pushouts and $\sharp$ is a pullback. 


\section{Theorem 27 (Concurrency Theorem).}

1. Given an $X$-dependent derivation $C \longrightarrow \triangleright_{p_{1}, f_{1}} D_{1} \longrightarrow \triangleright_{p_{2}, f_{2}} D$ there exists an $X$-concurrent derivation $C \longrightarrow \triangleright_{p_{1} ; x} p_{2} D$

2. Given an $X$-concurrent derivation $C \longrightarrow \triangleright_{p_{1} ; x} p_{2} D$, there exists an $X$ dependent derivation $C \longrightarrow \triangleright_{p_{1}, f_{1}} D_{1} \longrightarrow \triangleright_{p_{2}, f_{2}} D$.

\section{Relation with High-Level Replacement Categories}

High-level replacement categories [91011] or HLR-categories encompass several attempts to isolate general categorical axioms which lead to categories in which one can define double-pushout graph rewriting and prove useful theorems such as the local Church-Rosser theorem and the concurrency theorem.

HLR-categories usually have axioms which are parametrised over an arbitrary class of morphisms $\mathcal{M}$. Here we give a simplified version of the definition which appears in 9]. The simplification is that we take $\mathcal{M}$ to be the class of monomorphisms: we justify this by noting that this is the case in the majority of examples.

Definition 28 (HLR-categories) A category $\mathbf{S}$ is an HLR-category if it satisfies the following axioms:

1. pairs $C \leftarrow A \rightarrow B$ with at least one of the arrows mono have a pushout;

2. pairs $B \rightarrow D \leftarrow C$ with both morphisms mono have pullbacks;

3. monos are preserved by pushout;

4. finite coproducts exist;

5. pushouts of monos are pullbacks;

6. pushout-pullback decomposition holds: that is, given a diagram

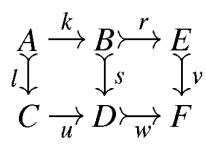

if the marked morphisms are mono, the whole rectangle is a pushout and the right square is a pullback, then the left square is a pushout.

Lemma 29 Any adhesive category with an initial object is an HLR-category.

Proof. This follows immediately from Lemmas 12, 13, and 16,

The axioms listed above are enough to prove the local Church-Rosser theorem (cf. Theorem [23), but not the concurrency theorem (cf. Theorem [27). To prove the latter, extra axioms had to be introduced in [11], such as the conclusion of the following lemma. Interestingly, it is almost the dual of the main axiom of adhesive categories. 
Lemma 30 (Cube-pushout-pullback-lemma [11]) Given a cube in which all arrows in the top and bottom faces are mono, if the top face is a pullback and the front faces are pushouts, then the bottom face is a pullback if and only if the back faces are pushouts.

Proof. Since the front faces are pushouts along monomorphisms, they are also pullbacks.

If the bottom face is a pullback, then the back faces are pushouts by stability of the pushouts on the front faces. Suppose conversely that the back faces are pushouts; since they are pushouts along monomorphisms, they are also pullbacks. One now simply "rotates the cube": since the front right and back left faces are pushouts, and the top and back right faces are pullbacks, it follows by adhesiveness that the bottom square is a pullback.

An HLR-category which has the conclusion of Lemma 30 as an additional axiom is sometimes referred to as an HLR2-category [11]. It is immediate, therefore, that any adhesive category with an initial object is an HLR2-category.

The strongest axiom system for general rewriting is enjoyed by the so-called HLR2*-categories [11. These are HLR2-categories which, additionally, have the conclusion of Lemma 15 as an axiom, that is, pushout complements of monos are, if they exist, unique up to isomorphism. Finally, they satisfy an axiom known as the twisted-triple-pushout-condition. We believe that this axiom does not hold in an arbitrary adhesive category, although it does hold, for instance, in any topos. Indeed, it is possible to extend the definition of adhesive categories in a natural way so that the twisted-triple-pushout-condition holds [16].

\section{Conclusions and Future Work}

We introduced the notions of van Kampen (VK) square and adhesive category. VK squares are "well-behaved pushouts", and a category is adhesive when pushouts along monos are VK. Adhesive categories are closely related to extensive categories.

Double-pushout ( $\mathrm{d}-\mathrm{p})$ rewriting can be defined in an arbitrary adhesive category. We introduced adhesive grammars, which are adhesive categories with a set of linear productions. Adhesive grammars have sufficient structure for the development of a rich rewriting theory. In particular, we proved the local ChurchRosser and the so-called concurrency theorem within the setting of adhesive grammars. We have also shown that adhesive categories satisfy many of the axioms 911 which were proposed in order to prove these theorems. Thus, we have arrived at a class of categories which supports such a theory of d-p rewriting, however, we believe that adhesive categories are mathematically elegant and less ad-hoc than previous proposals.

In order to back this claim and to further develop the theory of adhesive categories, we have demonstrated a number of useful properties. For instance, subobject union is formed as a pushout over the intersection, and subobject intersection distributes over subobject union. We have provided some closure properties which allow the construction of new adhesive categories from old. Any 
elementary topos is adhesive, but there are examples of adhesive categories which are not toposes. Adhesive categories include many well-known notions of graph structures used in computer science and are instances of HLR2-categories [11.

We believe that adhesive categories will be useful in the development of specific graphical models of computation and the development of semantic techniques for reasoning about such models. The rewriting theory needs to be developed further, with, for example, the construction of canonical dependency relations from derivations [11. A related task is to clarify the relationship of adhesive categories and the HLR $2 *$-categories [11].

Another possible direction for future work is to examine whether adhesive categories have enough structure so that groupoidal relative pushouts 21] can be constructed in cospan bicategories over adhesive categories. Such cospan bicategories provide a way of understanding graphs in a modular fashion and will provide a general class of models which should include bigraphs [18] as examples. A further question to be resolved is whether demanding the good behaviour of pushouts only along some class of monomorphisms will result in further interesting categories.

Acknowledgement. The second author would like to thank Vladimiro Sassone for many discussions in the early stages of this project. Thanks also go to Marco Carbone, Mogens Nielsen, Paolo Oliva, Mikkel Nygaard Ravn and the anonymous referees for reading early drafts and providing many valuable comments and suggestions.

\section{References}

1. P. Baldan, A. Corradini, H. Ehrig, M. Löwe, U. Montanari, and F. Rossi. Concurrent semantics of algebraic graph transformations. In H. Ehrig, H.-J. Kreowski, U. Montanari, and G. Rozenberg, editors, Handbook of Graph Grammars and Computing by Graph Transformation, volume 3, chapter 3, pages 107-187. World Scientific, 1999.

2. R. Brown and G. Janelidze. Van Kampen theorems for categories of covering morphisms in lextensive categories. J. Pure Appl. Algebra, 119:255-263, 1997.

3. A. Carboni, S. Lack, and R. F. C. Walters. Introduction to extensive and distributive categories. Journal of Pure and Applied Algebra, 84(2):145-158, February 1993.

4. L. Cardelli. Bitonal membrane systems. Draft, 2003.

5. A. Corradini, H. Ehrig, R. Heckel, M. Lowe, U. Montanari, and F. Rossi. Algebraic approaches to graph transformation part i: Basic concepts and double pushout approach. In G. Rozenberg, editor, Handbook of Graph Grammars and Computing by Graph Transformation, volume 1, pages 162-245. World Scientific, 1997.

6. V. Danos and C. Laneve. Graphs for core molecular biology. In International Workshop on Computational Methods in Systems Biology, CMSB '03, 2003.

7. H. Ehrig. Introduction to the algebraic theory of graph grammars. In 1st Int. Workshop on Graph Grammars, Lecture Notes in Computer Science LNCS 73, pages 1-69. Springer Verlag, 1979. 
8. H. Ehrig, G. Engels, H.-J. Kreowski, and G. Rozenberg, editors. Handbook of Graph Grammars and Computing by Graph Transformation, Volume 2: Applications, Languages and Tools. World Scientific, 1999.

9. H. Ehrig, M. Gajewsky, and F. Parisi-Presicce. High-level replacement systems with applications to algebraic specifications and Petri Nets. In H. Ehrig, H.-J. Kreowsky, U. Montanari, and G. Rozenberg, editors, Handbook of Graph Grammars and Computing by Graph Transformation, volume 3, chapter 6, pages 341-400. World Scientific, 1999.

10. H. Ehrig, A. Habel, H.-J. Kreowski, and F. Parisi-Presicce. From graph grammars to high level replacement systems. In 4th Int. Workshop on Graph Grammars and their Application to Computer Science, volume 532 of Lecture Notes in Computer Science, pages 269-291. Springer Verlag, 1991.

11. H. Ehrig, A. Habel, H.-J. Kreowski, and F. Parisi-Presicce. Parallelism and concurrency in high-level replacement systems. Math. Struct. in Comp. Science, 1, 1991.

12. H. Ehrig, H.-J. Kreowski, U. Montanari, and G. Rozenberg, editors. Handbook of Graph Grammars and Computing by Graph Transformation, Volume 3: Concurrency, Parallelism and Distribution. World Scientific, 1999.

13. H. Ehrig, M. Pfender, and H.J. Schneider. Graph-grammars: an algebraic approach. In IEEE Conf. on Automata and Switching Theory, pages 167-180, 1973.

14. H.-J. Kreowski. Transformations of derivation sequences in graph grammars. In Lecture Notes in Computer Science, volume 56, pages 275-286, 1977.

15. S. Lack and P. Sobociński. Adhesive categories. Technical Report RS-03-31, BRICS, October 2003.

16. S. Lack and P. Sobociński. Van Kampen squares and adhesive categories. In preparation, 2003.

17. J. Lambek and P. J. Scott. Introduction to higher order categorical logic, volume 7 of Cambridge studies in advanced mathematics. Cambridge University Press, 1986.

18. R. Milner. Bigraphical reactive systems: Basic theory. Technical Report 523, Computer Laboratory, University of Cambridge, 2001.

19. U. Montanari, M. Pistore, and F. Rossi. Modelling concurrent, mobile and coordinated systems via graph transformations. In H. Ehrig, H.-J. Kreowski, U. Montanari, and G. Rozenberg, editors, Handbook of Graph Grammars and Computing by Graph Transformation, volume 3, chapter 4, pages 189-268. World Scientific, 1999.

20. G. Rozenberg, editor. Handbook of Graph Grammars and Computing by Graph Transformation, Volume 1: Foundations. World Scientific, 1997.

21. V. Sassone and P. Sobociński. Deriving bisimulation congruences using 2categories. Nordic Journal of Computing, 10(2):163-183, 2003. 